\title{
Gemcitabine inhibits immune escape of pancreatic cancer by down regulating the soluble ULBP2 protein
}

\author{
Xiansheng Lin ${ }^{1,2, *}$, Mei Huang ${ }^{1,2, *}$, Fang $\mathrm{Xie}^{1}$, Hangcheng Zhou ${ }^{3}$, Ji Yang ${ }^{1}$, Qiang \\ Huang ${ }^{1,2}$ \\ ${ }^{1}$ Department of General Surgery, Affiliated Provincial Hospital of Anhui Medical University, Hefei, 230001, China \\ ${ }^{2}$ Anhui Province Key Laboratory of Hepatopancreatobiliary Surgery, Hefei, 230001, China \\ ${ }^{3}$ Department of Pathology, Affiliated Provincial Hospital of Anhui Medical University, Hefei, 230001, China \\ *These authors have contributed equally to this work \\ Correspondence to: Qiang Huang, email: ahslyyhq2016@163.com \\ Keywords: pancreatic cancer, gemcitabine, ULBP2, ADAM10, NK cells \\ Received: June 07, $2016 \quad$ Accepted: August 15, $2016 \quad$ Published: September 01, 2016
}

\section{ABSTRACT}

Due to early onset of local invasion and distant metastasis, pancreatic cancer is the most lethal human malignant tumor, with a $\mathbf{5}$ year survival rate of less than $5 \%$. As a effective chemotherapy drug for pancreatic cancer patients, gemcitabine is reported to inhibit cell proliferation as a nucleotide analog. In this study, we investigated the role of gemcitabine in immune regulation of pancreatic cancer. Our data showed that the level of soluble ULBP2 (sULBP2), a ligand of NKG2D receptor, decreased in the supernatants of pancreatic cancer cell lines when gemcitabine was added, and SULBP2 level correlated with NK92 cells cytotoxicity to pancreatic cancer cell lines. Importantly, our data showed that gemcitabine promoted PANC-1 cells and MIA PaCa-2 immune evasion by reducing ADAM10 expression, a metalloproteinase involved in sULBP2 shedding from cell membrane. Knockdown of ADAM10 clearly downregulated sULBP2 levels in the culture supernatants and cells became more susceptible to NK92 cytotoxicity. Serum samples and tumor samples were obtained from 45 patients with pancreatic ductal adenocarcinoma (PDAC). Statistical analysis showed a significant correlation between the serum level of sULBP2 with ADAM10 expression in PDAC tissues. In conclusion, our data demostrated that gemcitabine inhibits ULBP2 ectodomain shedding through the suppression of ADAM10 and enhance NK cells cytotoxicity by NKG2D-ULBP2 interaction. The results extends our understanding of gemcitabine in the treatment of pancreatic cancer from cell proliferation inhibition to immune regulation.

\section{INTRODUCTION}

Pancreatic cancer has become the forth leading cause of cancer-related death worldwide, and is one of the most common malignant tumors $[1,2]$. Radical surgery remains the only option to cure pancreatic cancer, but few patients are diagnosed when surgical resection is feasible [3]. That is why the prognosis is poor, with similar mortality and incidence [4]. The average survival period after prognosis is no more than six months, whereas the overall 5-year survival rate is less than 5\%[5]. Therefore, it is urgent to develop novel therapeutic approaches to treat pancreatic cancer.
Gemcitabine is the only chemotherapy drug that has been demonstrated to show benefit in patients with pancreatic cancer [6]. Gemcitabine alone or in combination with other chemotherapy drug or radiation treatment may prolong survival of pancreatic cancer patients. It is reported to show an broad-spectrum anti-tumor effect for most solid tumors by destroying cell replication as a nucleotide analog [7]. However, little is reported about its regulation on cancer immunity. Gemcitabine may increase memory $\mathrm{T}$ cells and induce naïve $\mathrm{T}$ cell activation, and may enhance antitumor immunity induced by tumor vaccine $[8,9]$. To expand the application of gemcitabine in treatment of pancreatic cancer, its immunological impact needs to be evaluated. 
ULBP2, one of UL16-binding protein family, is a cell surface glycoprotein and functions as a stress-induced ligand for NKG2D receptor [10]. Various NKG2D ligands are shown to be upregulated by a range of primary tumors, including lung, kidney, prostate, breast and colon cancers [11-14]. Immune response induced by ULBP2-NKG2D may play an important role in the eradiation of tumors by $\mathrm{T}$ and/or NK cells.

In the present study, we investigated the correlation between the sULBP2 expression and gemcitabine, and found gemcitabine inhibit sULBP2 shedding from cell surface of pancreatic cancer cell lines, which protect pancreatic cancer from NK cells cytotoxicity. Furtherly, ADAM10 knockdown experiments demonstrated the essential roles of ADAM10 protease in the shedding of ULBP2. Gemcitabine showed anti-cancer effect by downregulating NK cells function via inhibition of ADAM10 expression and shedding of sULBP2, which broadens our previous understanding of gemcitabine in the treatment of pancreatic cancer.

\section{RESULTS}

\section{Gemcitabine inhibits shedding of ULBP2 in PANC-1 and MIA PACA-2 cells}

We cultured 2 pancreatic cancer cell lines, PANC-1 and MIA PACA-2 cells and analyzed culture supernatants from the two cell lines. The level of sULBP2 decreased after gemcitabine was added to the culture medium of PANC-1 and MIA PACA-2 cells (Figure 1a). Gemcitabine was found to inhibit shedding of ULBP2 at concentrations of $\leq 2 \mu \mathrm{mol} / \mathrm{L}$. Based on this finding, gemcitabine with concentrations of $2 \mu \mathrm{mol} / 1$ was used to in the next experiments. FACS analysis showed ULBP2 was expressed on the cell surface on PANC-1 and MIA PACA-2 cells in the membrane form, and gemcitabine upregulated ULBP2 surface expression (Figure 1b).
Treatment with gemcitabine was observed to have markedly augmented membrane-bound ULBP2 expression and significantly decreased sULBP2 in PANC-1 cells and MIA PACA-2 cells.

\section{Gemcitabine enhances NK cells cytotoxicity to PANC-1 and MIA PACA-2 cells via ULBP2}

As a ligand of nature immune activating receptor NKG2D, ULBP2-NKG2D interaction may promote tumors immune evasion. We cultured NK92 cell lines and evaluated the cytotoxicity of NK92 cells to PANC1 or MIA PACA-2 cells using the CCK-8 assay. We cocultured NK92 cells and PANC-1 or MIA PACA-2 cells, with or without gemcitabine. Treatment with gemcitabine was shown to enhance NK cytotoxicity to PANC-1 and MIA PACA-2 cells, whereas SULBP2 protein decreased NK cytotocity to PANC-1 cells or MIA PACA-2 cells remarkably (Figure 2a, 2b). The results demonstrated gemcitabine may have effect on NK cells function to pancreatic cancer cells via NKG2D-ULBP2 pathway.

\section{Gemcitabine inhibits ULBP2 shedding via suppressing ADAM10 expression}

ADAM10 (a disintegrin and metalloproteinase 10) is reported to be responsible for the shedding of NKG2D ligands from the surface of various cell types via the proteolytic cleavage and release of the ectodomains of NKG2D ligands. To verify whether gemcitabine inhibits ULBP2 shedding through the suppression of ADAM10, we cultured PANC-1 cells and MIA PACA-2 cells with or without gemcitabine. Realtime PCR and western blot results showed that gemcitabine treatment downregulate ADAM10 expression in PANC-1 cells and MIA PACA-2 cells (Figure 3a). Then PANC-1 cells or MIA PACA-2 cells were transfected with siRNA against human ADAM10 or control siRNA. The downregulation of ADAM10 transcripts and
A

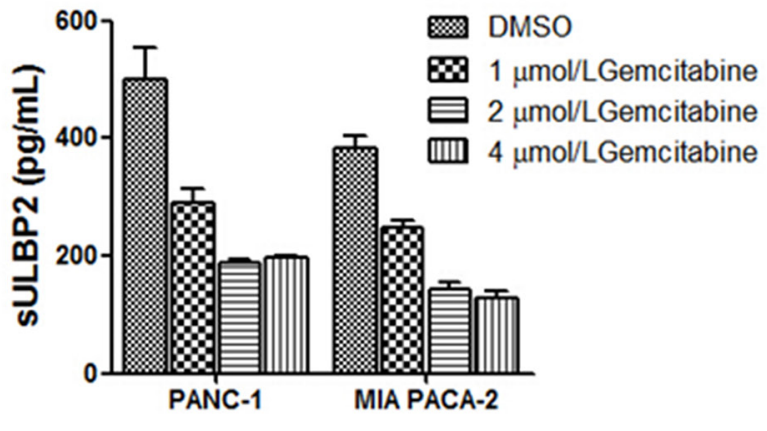

B

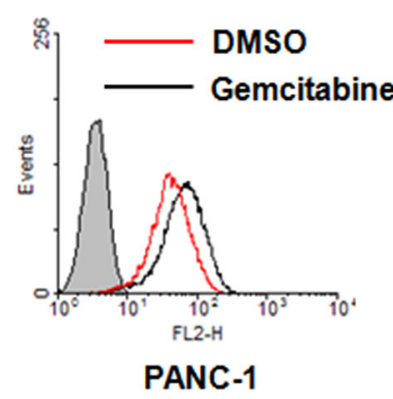

Figure 1: Gemcitabine inhibits shedding of ULBP2 in PANC-1 and MIA PACA-2 cells. A. PANC-1 cells and MIA PACA-2 cells were treated with different concentrations of gemcitabine or vehicle (DMSO) for $24 \mathrm{~h}$, and ULBP2 concentration was determinated by ELISA. B. Cells were treated with $2 \mu \mathrm{mol} / 1$ gemcitabine or vehicle (DMSO) for $24 \mathrm{~h}$ and membrane-bound ULBP2 expression was evaluated by flow cytometry. 

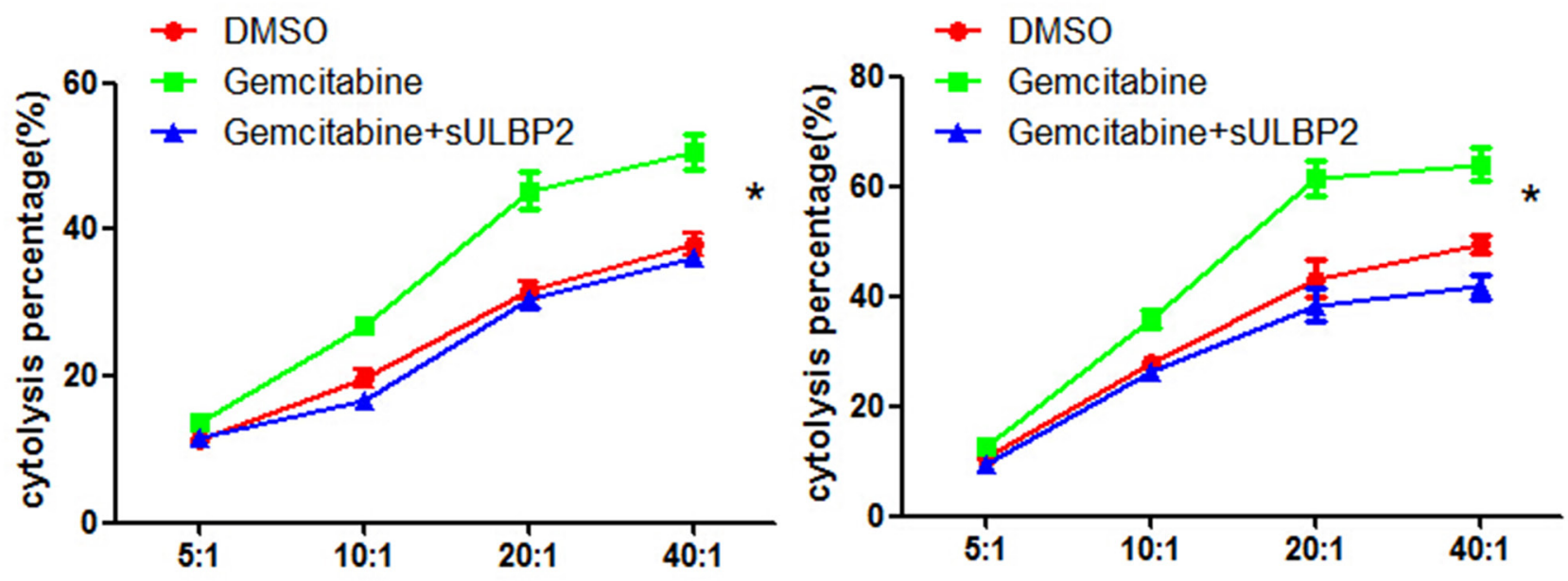

Figure 2: Gemcitabine enhances NK cells cytotoxicity to PANC-1 and MIA PACA-2 cells via sULBP2. The CCK-8 assay was used to determine the cytotoxicity of NK92 cells to PANC-1 cells A. and MIA-PACA2 cells B. Cells were treated with $2 \mu \mathrm{mol} / 1$ of gemcitabine (green) or vehicle (DMSO, red) for $4 \mathrm{~h}$, and recombinant sULBP2 protein was added(blue).

A
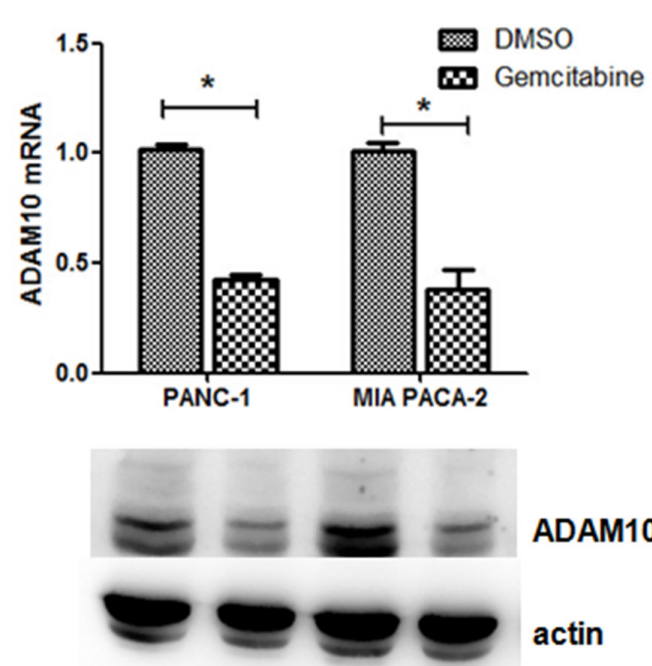

C

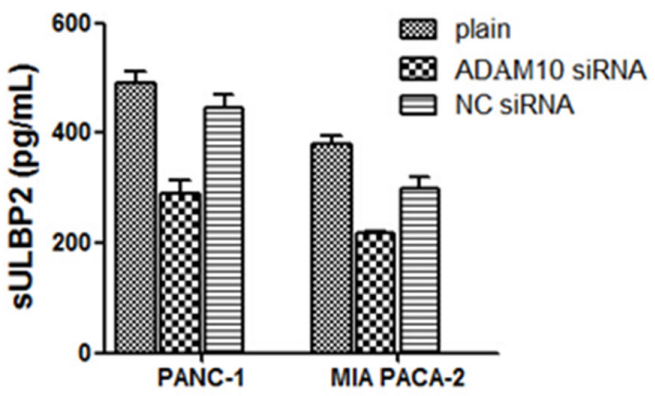

B
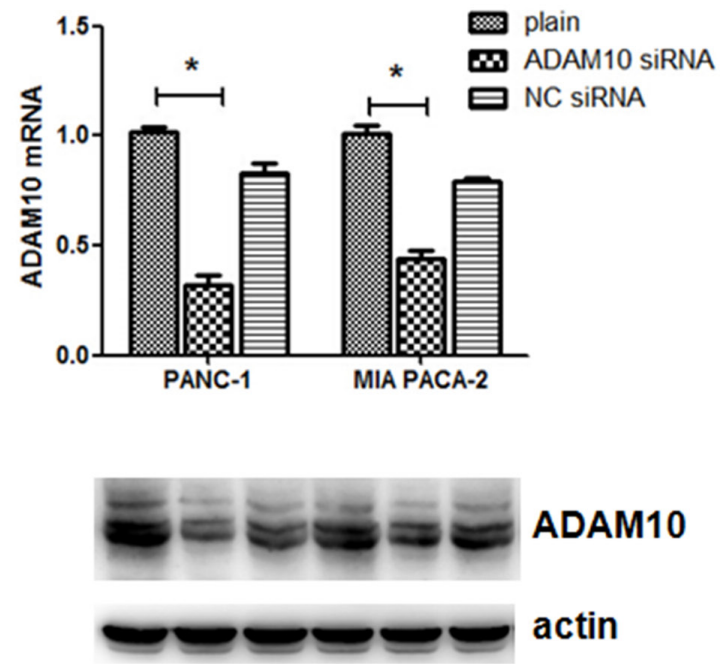

Figure 3: Gemcitabine-mediated shedding of ULBP2 is ADAM10-dependent. A. ADAM10 expression of PANC-1 and MIA PACA-2 cells was determined when $2 \mu \mathrm{mol} / 1$ gemcitabine was added into cell culture. B. Cells were transfected with ADAM10 siRNA or control siRNA for $48 \mathrm{~h}$ and the expression of mRNA and protein of ADAM10 was evaluated by Q-PCR and western blot. C. sULBP2 in the culture supernatant was evaluated by ELISA. $* \mathrm{P}<0.05$. 
ADAM10 protein was monitored by real-time RT-PCR and by western blotting, respectively (Figure $3 \mathrm{~b}$ ). No difference was noted in proliferation between the control and ADAM10 knockdown cells (data not shown). Knockdown of ADAM10 for PANC- 1 cells and MIA PACA- 1 cells resulted in $40.95 \%$ and $42.7 \%$ reduction of sULBP2 levels in the culture medium, respectively (Figure $3 \mathrm{c}$ ). Taken together, these results suggest that gemcitabine inhibits ULBP2 shedding in PANC-1 cells and MIA PACA-1 cells by downregulating the expression of ADAM10.

\section{sULBP2 level is correlated with poor prognosis and ADAM10 expression}

We next investigated serum levels of ULBP2 by ELISA assay in 45 PDAC patients (Supplementary Table $\mathrm{S} 1)$ and 45 healthy individuals, and the sULBP2 levels of PDAC patients were significantly higher $(p<0.001)$ than in healthy controls (data not shown). Based on ROC analysis of PDAC patients and healthy controls, the cutoff value of $16.11 \mathrm{pg} / \mathrm{ml}$ was used to divide the serum sample into groups that were negative or positive for sULBP2 (Supplementary Figure S1). The expression of ADAM10 was determined using immunohistochemical analysis, which showed that ADAM10 staining was mainly located in the cytoplasm of tumor cells with varying staining intensity (Figure 4). The clinical and pathological characteristics of the pancreatic cancer patients are presented in Table 1. A significant difference was observed in the serum ULBP2 levels with regard to the CA199 levels ( $\mathrm{p}=0.013$ ), lymph node metastasis $(\mathrm{p}=0.009)$ and overall survival $(\mathrm{p}=0.045)$ (Figure 5). There was no significant correlation between the ADAM10 expression with age, gender, tumor size, perineural invasion, or lymph node metastasis ( $\mathrm{P}>0.05$, respectively). Serum ULBP2 was found to be positively correlate with ADAM10 expression. The results indirectly confirmed that the effect of gemcitabine on pancreatic cancer may be related to ADAM10 and ULBP2.

\section{DISCUSSION}

Gemcitabine is the standard chemotherapy regimen for the treatment of advanced pancreatic cancer [15]. It is a nucleoside analogue, which exerts its anti-tumor effect through a variety of mechanisms, mainly via inhibition of DNA replication and mask of DNA chain termination [16]. Therefore, it prevents the DNA synthesis and DNA repair, causes the cell to enter apoptotic pathway. However, its role in immune response have not be fully documented. Our results demonstrated that gemcitabine inhibit ULBP2 ectodomain shedding through down-regulation of ADAM10 expression in PANC-1 and MIA PACA-2 cell lines. Decrease of sULBP2 and increase of membranebound ULBP2 thus promote NK cells activation and may improve the antitumor effect against pancreatic cancer, which will be further confirmed by studies on tissues of pancreatic cancer patients.

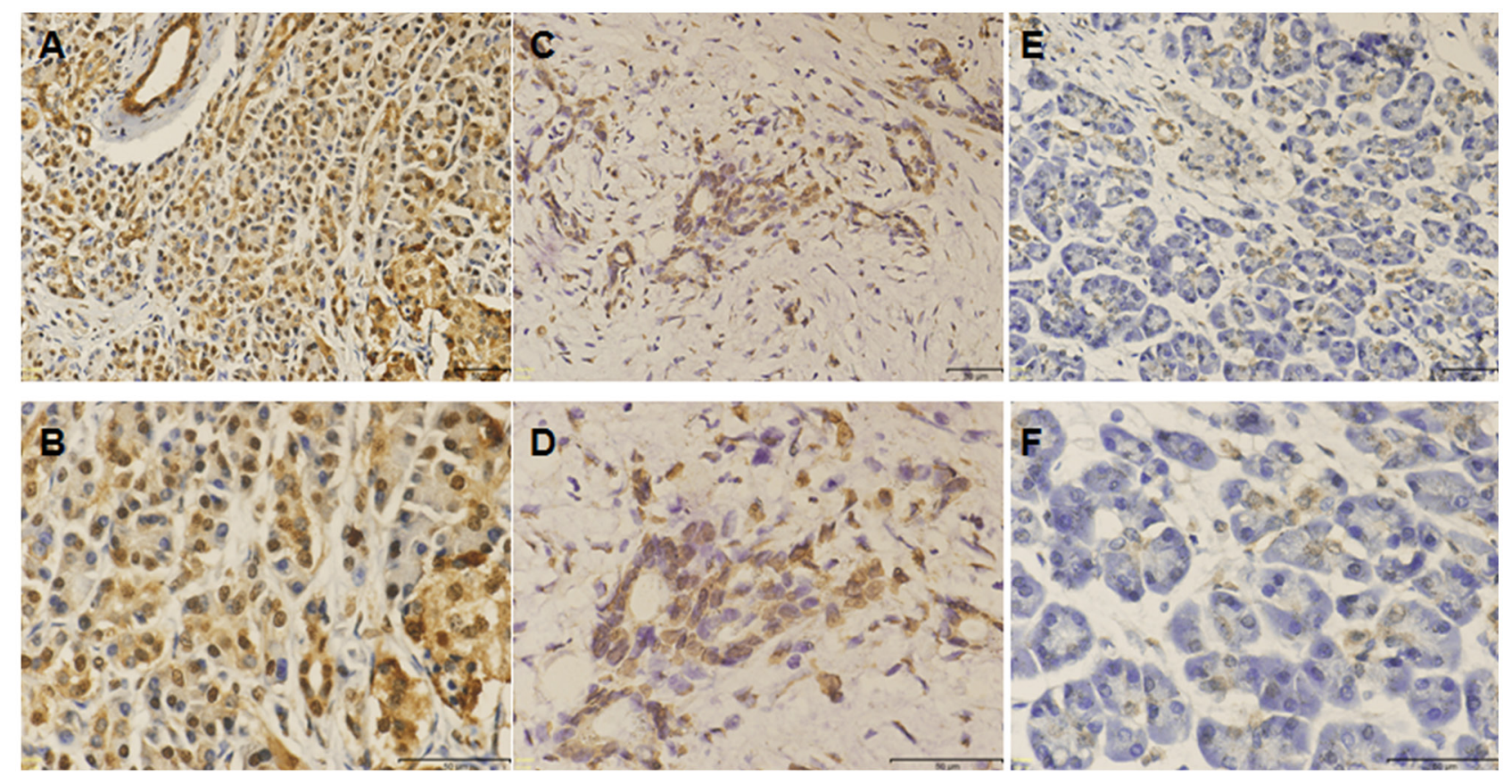

Figure 4: Immunohistochemical staining for ADAM10. The ADAM10 were principally localized in cytoplasm of tumor cells with varying staining intensity. A. High expression of ADAM10 (200x). B. High expression of ADAM10 (400x). C. Low expression of ADAM10 (200x). D. Low expression of ADAM10 (400x). E. Negative ADAM10 expression (200x). F. Partial enlargement of ADAM10 staining with the magnifying power of 400x. 
Table 1: Correlation between ULBP2 and ADAM10 expression and clinicopathological characteristics

\begin{tabular}{|c|c|c|c|c|c|c|c|}
\hline & \multirow[t]{2}{*}{ n } & \multicolumn{2}{|c|}{ ADAM10 staining } & \multirow[t]{2}{*}{$\mathbf{p}$} & \multicolumn{2}{|c|}{$\begin{array}{l}\text { Serum ULBP2 (pg/ } \\
\text { ml) }\end{array}$} & \multirow[t]{2}{*}{$\mathbf{p}$} \\
\hline & & $-/+$ & $++/+++$ & & $<16.11$ & $\geq 16.11$ & \\
\hline All & 45 & 6 & 39 & & 4 & 41 & \\
\hline \multicolumn{8}{|l|}{ Gender } \\
\hline female & 16 & 0 & 16 & 0.171 & 0 & 16 & 0.261 \\
\hline male & 29 & 6 & 23 & & 4 & 25 & \\
\hline \multicolumn{8}{|l|}{ Age } \\
\hline$<60$ & 19 & 2 & 17 & 0.141 & 0 & 19 & 0.238 \\
\hline$\geq 60$ & 26 & 4 & 22 & & 4 & 22 & \\
\hline \multicolumn{8}{|l|}{ CA19-9 } \\
\hline$<37 \mathrm{U} / \mathrm{ml}$ & 10 & 0 & 10 & 0.138 & 0 & 10 & 0.013 \\
\hline$\geq 37 \mathrm{U} / \mathrm{ml}$ & 35 & 6 & 29 & & 4 & 31 & \\
\hline \multicolumn{8}{|c|}{ Tumor size (cm) } \\
\hline$\leq 2$ & 5 & 1 & 4 & 0.214 & 1 & 4 & 0.184 \\
\hline$>2$ & 40 & 5 & 35 & & 3 & 37 & \\
\hline \multicolumn{8}{|c|}{ Tumor location } \\
\hline Head & 34 & 4 & 30 & 0.024 & 2 & 32 & 0.100 \\
\hline Body/tail & 11 & 2 & 9 & & 2 & 9 & \\
\hline \multicolumn{8}{|c|}{ Histological grade } \\
\hline Well-mod & 25 & 4 & 21 & 0.099 & 2 & 23 & 0.092 \\
\hline poor & 20 & 2 & 18 & & 2 & 18 & \\
\hline \multicolumn{8}{|l|}{ TNM stage } \\
\hline I-II & 34 & 5 & 29 & 0.078 & 3 & 31 & 0.059 \\
\hline III-IV & 11 & 1 & 10 & & 1 & 10 & \\
\hline \multicolumn{8}{|c|}{ Perineural invasion } \\
\hline present & 20 & 4 & 16 & 0.079 & 3 & 17 & 0.208 \\
\hline absent & 25 & 2 & 23 & & 1 & 24 & \\
\hline \multicolumn{8}{|l|}{ LNM } \\
\hline present & 17 & 1 & 16 & 0.098 & 1 & 16 & 0.009 \\
\hline absent & 28 & 5 & 23 & & 3 & 25 & \\
\hline
\end{tabular}

In the present study, the level of serum ULBP2 was examined in 45 PDAC patients using ELISA. sULBP2 was found to be over-expressed in sera of pancreatic cancer patient compared with healthy individuals. Moreover, a significant difference was noted in the serum ULBP2 level with regard to the CA199 levels and lymph node metastasis. Serum levels of ULBP2 in pancreatic cancer patients were also found to correlate significantly with shorter overall survival and poor prognosis. ULBP2 shedding is thought to be a principal mechanism by which tumor cells escape from NKG2D-mediated immune surveillance in pancreatic cancer. Therefore, ULBP2 is hypothesized to be associated with the malignant transformation of pancreatic cancer.

ADAM10 is one of the the ADAM family of disintegrin proteinasesm, and mediates proteolytic release of ectodomains of transmembrane proteins from the cell surface, including cytokines, growth factors and cell adhesion molecules [17, 18]. The present study demonstrated that ADAM10 expression 


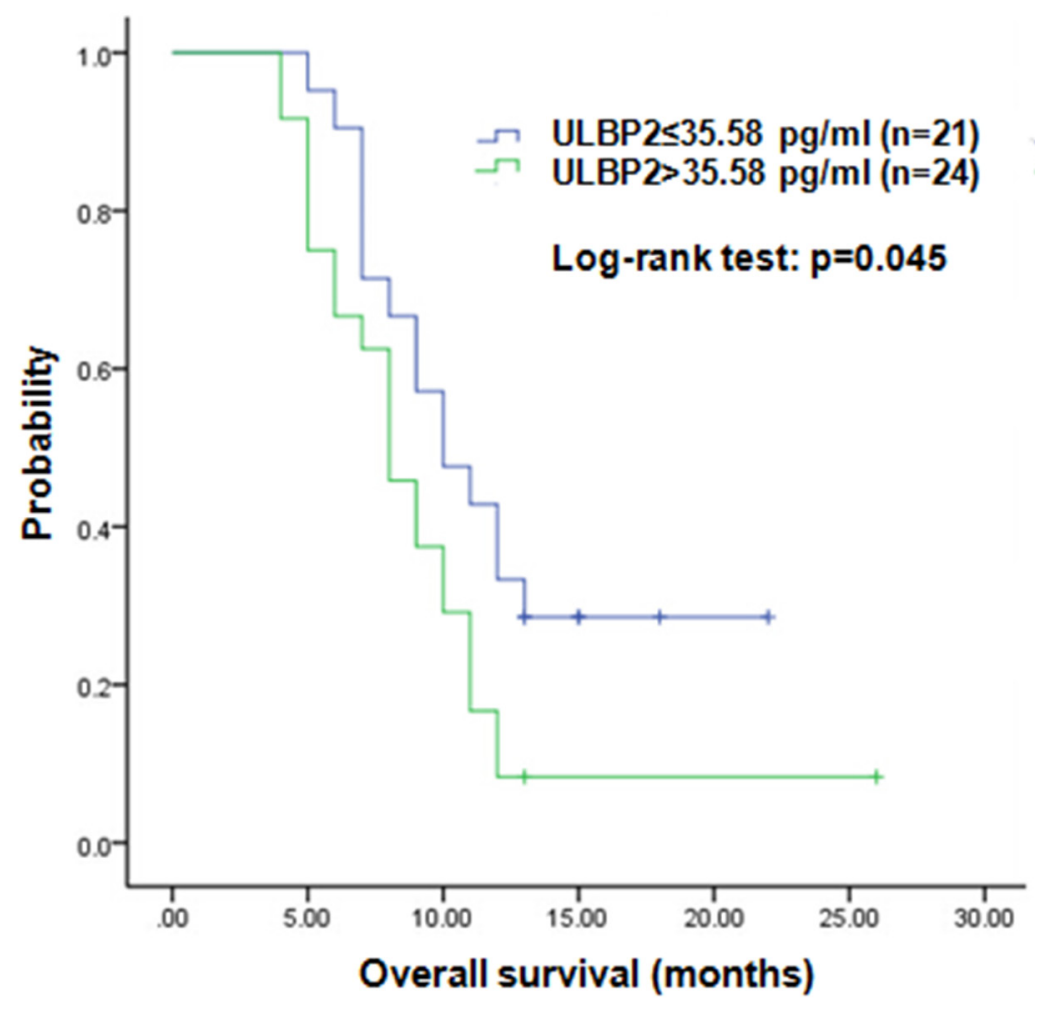

Figure 5: Kaplan-Meier analysis of overall survival. sULBP2 low was defined as sULBP2 $\leq 35.58$ pg/ml (median). sULBP2 high was defined as sULBP2 $>35.58 \mathrm{pg} / \mathrm{ml}$. The P-value was determined using the log-rank test.

is significantly lower in gemcitabine treated pancreatic cell lines. Meanwhile, serum levels of ULBP2 was positively correlated with ADAM10 expression shown by immunohistochemistry, suggesting that gemcitabine may exerts its anti-tumor effect through inhibition of ADAM10 mediated ULBP2 shedding and corresponding enhanced NKG2D-mediated tumor elimination.

In this study, the siRNA mediated ADAM10 knockdown was found to decrease the release of sULBP2, which proved the essential role for the cleavage of SULBP2. The observations suggest that ADAM10 may serve as a potential therapeutic target for treatment of pancreatic cancer patients who are not sensitive to gemcitabine.

In conclusion, gemcitabine has been demonstrated not only to inhibit cell proliferation, but also to enhance the immune response of pancreatic cancer cells by enhancing the activity of NK cells. The present study sheds light on previously unrecognized effects of gemcitabine on tumor immune response, modulating ADAM10 and ULBP2 shedding, thus suggesting its use in chemoimmunotherapy against human pancreatic cancer.

\section{MATERIALS AND METHODS}

\section{Subjects and samples}

Forty-five patients with pancreatic cancer were enrolled between January 2013 and Dec 2015. The patients were surgically treated in the Affiliated Provincial Hospital of Anhui Medical University(Hefei, China). The samples of cancer tissue were obtained during surgery, and then fixed in $10 \%$ formalin solution and embedded in paraffin. The diagnosis of the samples was confirmed histopathologically. Informed consent was obtained from each patient, and the study protocol was approved by the Research Ethics Committee of Anhui Provincial Hospital. Follow-up data were collected from all 45 patients, and the mean followup period in the present investigation was 9.8 (range 4-26) months. Overall survival (OS) time was calculated from the date of surgery to the date of death or last observation.

\section{Cell lines}

The PANC-1, MIA PaCa-2 pancreatic cancer cell lines and NK92 cell lines were obtained from Shanghai cell bank (Shanghai, China). PANC-1 cells were maintained in DMEM (GIBCO) with $10 \%$ heat inactivated fetal bovine serum(FBS), 50 units $/ \mathrm{mL}$ penicillin, and 50 units/mL streptomycin. MIA PaCa-2 cells were cultured in DMEM supplemented with 10\% FBS, 2.5\% horse serum, $1 \%$ sodium pyruvate $100 \mathrm{mM}$ solution (Invitrogen), 50 units/mL penicillin, and 50 units/mL streptomycin. NK92 cells were maintained in $\alpha$-MEM (GIBCO) with $10 \% \mathrm{FBS}$, $100 \mathrm{U} / \mathrm{mL}$ recombinant human IL-2 (rhIL-2; PeproTech), 50 units $/ \mathrm{mL}$ penicillin, and 50 units $/ \mathrm{mL}$ streptomycin. All cells were cultured in humidified air with $5 \% \mathrm{CO}_{2}$ at $37^{\circ} \mathrm{C}$. 


\section{Quantitative real-time PCR}

Total RNA samples was extracted by using Trizol according to the manufacturer's protocol. Quantitative PCR was performed using SYBR Premix Ex Taq (Takara). The primer sequences used were as follows: ADAM10 forward, 5'- TTGGGAAGATGGTAGCTTGG -3'; reverse, 5'- CACATATTCCTCCAGAGCTTCC-3'; GAPDH mRNA served as internal control.

cDNA was amplified $\left(40\right.$ cycles, $95^{\circ} \mathrm{C}$ for $10 \mathrm{sec}$, $60^{\circ} \mathrm{C}$ for $30 \mathrm{sec}, 72$ for $30 \mathrm{sec}$ ) with an ABI StepOnePlus according to the manufacturer's instructions. A melting curve analysis was performed to monitor PCR-product purity, and relative gene expression data were analyzed using the $2-\Delta \Delta \mathrm{Ct}$ method.

\section{Western blot}

PANC-1 cells and MIA PACA-2 cells were lysed with lysis buffer (Beyotime Institute of Biotechnology, China), and protein concentration was subsequently estimated by the bicinchoninic acid protein assay. Then, equal amounts of protein samples were separated on $10 \%$ sodium dodecyl sulfate polyacrylamide gel electrophoresis gels and transferred onto polyvinylidene difluoride membranes (Millipore, USA). After being blocked with $5 \%$ nonfat milk, membranes were incubated overnight at $4{ }^{\circ} \mathrm{C}$ with rabbit primary antibodies against human ADAM10 (Boster, China) and human actin (Zhongshan Golden Bridge Biotechnology, China). After being washed with Tris-buffered saline $/ 0.1 \%$ Tween three times, membranes were incubated with secondary antibodies for 1 hours at room temperature. Immunoreactive bands were visualized using the enhanced chemiluminescence (ECL; Pierce,USA) kit. The specific protein bands were captured and visualized using FUSION FX5 (Vilber Lourmat).

\section{ELISA assay}

Blood samples were procured from the pancreatic cancer patients with informed consent according to the institutional guidelines. Serum samples were centrifuged at $1500 \mathrm{~g}$ for $10 \mathrm{~min}$ and the sera were stored at $-80^{\circ} \mathrm{C}$ until usage. Supernatants of the cell lines were harvested at $72 \mathrm{hr}$. For the determination of ULBP2 levels, commercial DuoSet ELISA kits (R\&D Systems) was used, and assays were performed following the manufacturer's instructions. The data were analyzed with Origin 8.0 software.

\section{siRNA-mediated knockdown}

The small interfering RNA (siRNA) method was used to knockdown ADAM10. Chemically synthesized ADAM10 siRNA and negative control siRNA were purchased from Genechem (Shanghai, China). Lipofectamine 2000 (Invitrogen) was used for transfection following the manufacture's instructions. The siRNAs used were: ADAM10, 5'-AACCCAGCTGTCACCCTCGAA-3'; negative control, 5'-TTCGAGGGTGACAGCTGGGTT-3'.

\section{Flow cytometric analysis}

For the detection of membrane-bound ULBP2, cells were incubated with phycoerythrin (PE)-mouse anti ULBP2 antibody (FAB1298P, R\&D systems) and then subjected to flow cytometry. Flow cytometry was performed using a FACSCalibur, and data were analyzed using WinMDI2.9 software.

\section{CCK-8 assay}

The CCK-8 (cell counting kit-8) assay was used to determine the cytotoxicity of NK92 cells. PANC-1 or MIA PACA-2 cells were used as target cells. Target cells were seeded in 96-well plates, with $1 * 104$ cells in each well, with or without gemcitabine. NK92 cells were incubated with target cells at effector/target ratios of 1:1, 1:5, 1:10 or 1:25 for $4 \mathrm{hr}$. A $20 \mu \mathrm{L}$ aliquot of enhanced CCK8 solution $(5 \mathrm{mg} / \mathrm{ml}$ in PBS) was then added to each well. Following $1 \mathrm{hr}$ of incubation, absorbance was measured on an ELISA reader at a test wavelength of $450 \mathrm{~nm}$. The percentage of cytolysis was calculated as [1-(Effect with Target cells)/ Target] $\times 100$.

\section{Immunohistochemistry}

Serial tissue sections (4 $\mu \mathrm{m}$ thick) were deparaffinized with xylene, rehydrated, and subjected to microwave antigen retrieval in citrate buffer $(\mathrm{pH}$ 6.0) for 20 minutes. Endogenous peroxidase activity was quenched by $3 \%$ hydrogen peroxide for 10 minutes. Afterward, the sections were incubated at $4^{\circ} \mathrm{C}$ overnight with monoclonal antibody (anti-ADAM10; Abcam) in a humidified chamber. After being washed, the sections were incubated for 30 minutes with horseradish peroxidase-conjugated secondary antibody (Zhongshan Golden Bridge Biotechnology). Immunoreactivity was visualized with chromogen 3,3'diaminobenzidine. Finally, all slides were counterstained with ematoxylin, dehydrated, and mounted. Tumor expression of ADAM10 was semiquantitatively evaluated using a previously reported method, and the percentage of staining cell scores $(0$, no staining; $1,10 \% ; 2,10 \%-$ $30 \% ; 3,>30 \%)$ and the staining intensity scores $(0$, negative; 1 , weak; 2 , moderate; 3 , strong) were summed. The final scores were ranged as following: total score $<$ 3 was defined as negative (-), 3-6 as"“+”, 6-9 as"++", and 9-12 as "+++". All sections were assessed by two pathologists who were blinded to clinical data.

\section{Statistical analysis}

Data were expressed as the mean $\pm \mathrm{SD}$. The means between groups were analyzed using the Mann-Whitney 
U test. All statistical analyses were performed using SPSS 16.00 statistical analysis software (SPSS Inc). Differences were considered statistically significant if $p<0.05$.

\section{ACKNOWLEDGMENTS}

We would like to thank to all of our colleagues for their constructive suggestions on the current study.

\section{CONFLICTS OF INTEREST}

The authors have decalared that no competing interests exist.

\section{FINANCIAL SUPPORT}

This study was supported by Natural Science Foundation of Anhui Province(Grant No. 1608085QH197;1408085QH186) and the Programs for Science and Technology Development of Anhui Province in 2015(No.1506c085018).

\section{REFERENCES}

1. Jemal A, Bray F, Center MM, Ferlay J, Ward E, Forman D. Global cancer statistics. CA Cancer J Clin. 2011; 61: 69-90.

2. Siegel R, Ma J, Zou Z, Jemal A. Cancer statistics, 2014. CA Cancer J Clin. 2014; 64: 9-29.

3. Hackert T, Buchler MW, Werner J. Surgical options in the management of pancreatic cancer. Minerva Chir. 2009; 64: 465-76.

4. Hidalgo M. Pancreatic cancer. N Engl J Med. 2010; 362: 1605-17.

5. Gong Z, Holly EA, Bracci PM. Survival in populationbased pancreatic cancer patients: San Francisco Bay area, 1995-1999. Am J Epidemiol. 2011; 174: 1373-81.

6. Lee HW, Chung MJ, Kang H, Choi H, Choi YJ, Lee KJ, Lee SW, Han SH, Kim JS, Song SY. Gemcitabine-induced hemolytic uremic syndrome in pancreatic cancer: a case report and review of the literature. Gut Liver. 2014; 8: $109-12$.

7. Toyota Y, Iwama H, Kato K, Tani J, Katsura A, Miyata M, Fujiwara S, Fujita K, Sakamoto T, Fujimori T, Okura R, Kobayashi K, Tadokoro T, et al. Mechanism of gemcitabineinduced suppression of human cholangiocellular carcinoma cell growth. Int J Oncol. 2015; 47: 1293-302.

8. Huang Q, Qu QX, Xie F, Hu JM, Chen YG, Zhang XG. Sensitization of $\mathrm{SiHa}$ cell to gemcitabine by CD40 activation and its overexpression in cervical carcinoma. Med Oncol. 2011; 28: 781-8.
9. Bauer C, Sterzik A, Bauernfeind F, Duewell P, Conrad C, Kiefl R, Endres S, Eigler A, Schnurr M, Dauer M. Concomitant gemcitabine therapy negatively affects DC vaccine-induced $\mathrm{CD} 8(+)$ T-cell and $\mathrm{B}$-cell responses but improves clinical efficacy in a murine pancreatic carcinoma model. Cancer Immunol Immunother. 2014; 63: 321-33.

10. Uhlenbrock F, Hagemann-Jensen M, Kehlet S, Andresen L, Pastorekova S, Skov S. The NKG2D ligand ULBP2 is specifically regulated through an invariant chain-dependent endosomal pathway. J Immunol. 2014; 193: 1654-65.

11. Okita R, Yukawa T, Nojima Y, Maeda A, Saisho S, Shimizu K, Nakata M. MHC class I chain-related molecule A and B expression is upregulated by cisplatin and associated with good prognosis in patients with non-small cell lung cancer. Cancer Immunol Immunother. 2016; 65: 499-509.

12. Rothe A, Jachimowicz RD, Borchmann S, Madlener M, Kessler J, Reiners KS, Sauer M, Hansen HP, Ullrich RT, Chatterjee S, Borchmann P, Yazaki P,Koslowsky TC, et al. The bispecific immunoligand ULBP2-aCEA redirects natural killer cells to tumor cells and reveals potent antitumor activity against colon carcinoma. Int J Cancer. 2014; 134: 2829-40.

13. de Kruijf EM, Sajet A, van Nes JG, Putter H, Smit VT, Eagle RA, Jafferji I, Trowsdale J, Liefers GJ, van de Velde CJ, Kuppen PJ. NKG2D ligand tumor expression and association with clinical outcome in early breast cancer patients: an observational study. BMC Cancer. 2012; 12: 24.

14. Lundholm M, Schroder $M$, Nagaeva O, Baranov V, Widmark A, Mincheva-Nilsson L, Wikström P. Prostate tumor-derived exosomes down-regulate NKG2D expression on natural killer cells and CD8+ T cells: mechanism of immune evasion. PLoS One. 2014; 9: e108925.

15. Heinemann V, Boeck S, Hinke A, Labianca R, Louvet C. Meta-analysis of randomized trials: evaluation of benefit from gemcitabine-based combination chemotherapy applied in advanced pancreatic cancer. BMC Cancer. 2008; 8: 82.

16. Abou-Alfa GK, Letourneau R, Harker G, Modiano M, Hurwitz H, Tchekmedyian NS, Feit K, Ackerman J, De Jager RL, Eckhardt SG, O'Reilly EM. Randomized phase III study of exatecan and gemcitabine compared with gemcitabine alone in untreated advanced pancreatic cancer. J Clin Oncol. 2006; 24: 4441-7.

17. Gibb DR, Saleem SJ, Chaimowitz NS, Mathews J, Conrad DH. The emergence of ADAM10 as a regulator of lymphocyte development and autoimmunity. Mol Immunol. 2011; 48: 1319-27.

18. Pabois A, Devalliere J, Quillard T, Coulon F, Gerard N, Laboisse C, Toquet C, Charreau B. The disintegrin and metalloproteinase ADAM10 mediates a canonical Notchdependent regulation of IL-6 through D114 in human endothelial cells. Biochem Pharmacol. 2014; 91: 510-21. 